\title{
5D Black Holes and Strings with Higher Derivatives
}

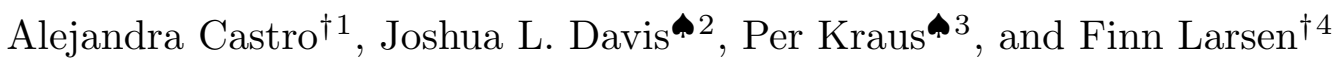 \\ $\dagger$ Department of Physics and Michigan Center for Theoretical Physics, \\ University of Michigan, Ann Arbor, MI 48109-1120, USA. \\ - Department of Physics and Astronomy, UCLA, \\ Los Angeles, CA 90095-1547, USA.
}

\begin{abstract}
We find asymptotically flat black hole and string solutions to 5D supergravity in the presence of higher derivative terms. In some cases, including the fundamental heterotic string solution, the higher derivative terms smooth out naked singularities into regular geometries carrying zero entropy. We also compute corrections to the entropy of 5D CalabiYau black holes, and discuss the relation to previous results.
\end{abstract}

March, 2007

\footnotetext{
1 aycastro@umich.edu

2 davis@physics.ucla.edu

3 pkraus@ucla.edu

4 larsenf@umich.edu
} 


\section{Introduction}

In this paper we find asymptotically flat solutions to 5D supergravity in the presence of four derivative terms. We obtain two types of solutions: magnetic string solutions, with near horizon geometry $\mathrm{AdS}_{3} \times S^{2}$; and electric particle solutions, with near horizon geometry $\mathrm{AdS}_{2} \times S^{3}$. These spacetimes are central to the study of black holes in string theory. Namely, the electric solutions represent 5D black holes, while the magnetic solutions yield $4 \mathrm{D}$ black holes upon compactification on a circle with momentum added.

The inclusion of higher derivative $R^{2}$ terms allows us to compute corrections to quantities such as the black hole entropy, and also to obtain smooth spacetimes in cases where the two derivative action yields a naked singularity. Via the AdS/CFT correspondence, this permits a more detailed comparison between the microscopic and macroscopic descriptions of black holes in string theory.

The program of including $R^{2}$ corrections in $4 \mathrm{D}$ supergravity has received much attention over the past few years [1,2, 3, 4, 5, 6, 7] (for reviews see [8,9, 10]). By contrast, the relevant 5D supergravity action was constructed only very recently, as the supersymmetric completion of a certain mixed gauge/gravitational Chern-Simons term [11]. The 5D theory can be thought of as arising from M-theory compactified on a Calabi-Yau threefold. The relevant higher derivative terms are determined by a combination of anomalies and supersymmetry.

Before proceeding, let us note some of the general advantages of working in a $5 \mathrm{D}$ setting. First, by compactifying one of our 5D directions on a circle (or more generally, a Taub-NUT fiber) we can reproduce all of the 4D solutions of interest [12,13, 14, 15, 16, 17]. On the other hand, by not compactifying we maintain access to solutions that are inherently five dimensional. One example is the standard 5D black hole [18, 19], which we'll refer to as the electric black hole. Another example is a straight fundamental heterotic string with zero momentum, which provides the simplest example of our magnetic solutions. To access these in the $4 \mathrm{D}$ description requires the auxiliary procedure of decompactifying a Taub-NUT fiber; here the description is simpler and more direct.

In this paper we restrict attention to spherically symmetric solutions to simplify the analysis. The generalization to spinning black holes and black rings will appear in subsequent work [20]. As we'll see, a very nice feature of the off-shell $R^{2}$ supergravity obtained in [11] is that the construction of BPS solutions is surprisingly simple. Indeed, the bulk of the analysis is entirely parallel to the two derivative case. The higher derivatives only manifest themselves towards the end of the construction, where they yield corrections to the standard special geometry relations, which are replaced by a more complicated non-linear differential equation.

In the magnetic string case our asymptotically flat solutions extend the near horizon attractor geometries we found in [21]. As described in [21], from the near horizon $\mathrm{AdS}_{3}$ 
solution we can read off the central charges of the dual CFT, and thereby confirm an earlier result based on anomalies and supersymmetry [6.22]. In the full solution the metric and matter fields exhibit oscillatory behavior of the same sort as found in [23,24]. As mentioned above, the simplest example is the single charge solution corresponding to a heterotic string with vanishing momentum (or, in the language of M-theory on $K 3 \times T^{2}$, an M5-brane wrapped on $K 3$ ). This provides an example of how $R^{2}$ corrections can replace a naked singularity with a smooth, zero entropy, geometry.

In the electric case, after constructing the black hole solutions we discuss their entropy. Given the near horizon $\mathrm{AdS}_{2}$ geometry, the computation of the Bekenstein-Hawking-Wald entropy reduces to evaluating a particular function at its extremum. The resulting entropy formula turns out to be extremely simple: expressed in terms of the charges, it takes the same form as in the two derivative case except that the charges are shifted by an amount proportional to the second Chern class of the Calabi-Yau. With a natural definition of horizon area, the formula $S=A / 4$ continues to hold.

We compare our corrected entropy formula to a previously conjectured result [25] based on the 4D-5D connection and the topological string free energy. The results precisely agree when expressed in terms of the electric potentials. Expressed instead in terms of the electric charges, there is a mismatch. Our entropy formula also agrees quantitatively with a correction found by Vafa in terms of a microscopic model of 5D black holes in M-theory on an elliptically fibred Calabi-Yau [26].

The remainder of this paper is organized as follows. In section 2 we review the construction of higher derivative terms using the off-shell formalism. In section 3 we find supersymmetric solutions of magnetic type, interpreted as string solutions. We establish that 5D strings interpolate smoothly between an $\mathrm{AdS}_{3} \times S^{2}$ near horizon geometry and an asymptotically flat region. In section 4 we find supersymmetric solutions of electric type. We discuss their near horizon behavior and we establish that they interpolate smoothly between an $\mathrm{AdS}_{2} \times S^{3}$ near horizon geometry and an asymptotically flat region. In section 5 we discuss the entropy of the 5D Calabi Yau black holes.

\section{5D supergravity with $R^{2}$ corrections}

We begin with a brief review of higher-derivative corrections to $N=2$ supergravity in five dimensions [11]. We use the superconformal formalism, developed in [27,28], which can be gauge-fixed to the familiar Poincare supergravity. Our conventions are summarized in appendix A.

\subsection{The supersymmetry transformations}

Before introducing the specific action that we analyze in this paper, let us briefly discuss the relevant supersymmetry multiplets. The irreducible Weyl multiplet contains 
the fields:

$$
e_{\mu}^{a}, \quad \psi_{\mu}, \quad V_{\mu}, b_{\mu}, v^{a b}, \quad \chi, \quad D
$$

The first two fields are the vielbein and gravitino. The $V_{\mu}$ is the vector boson associated with the gauging of the $S U(2)$ R-symmetry under which all fermionic variables and fields transform 5 , while $b_{\mu}$ is the gauge field of dilatational symmetry. We will ignore these gauge fields in the future, for they are gauged way when one reduces to Poincare supergravity. Finally are three auxiliary fields: an anti-symmetric tensor $v_{a b}$, the fermion $\chi$, and the scalar $D$. The vector multiplet consists of the gauge field $A_{\mu}^{I}$, the scalar $M^{I}$, the gaugino $\Omega^{I}$, and also a scalar $Y^{I}$, which will be gauged away.

Also of importance is the hypermultiplet. Although the hypers decouple from the physics we are developing, by gauge-fixing them we effectively couple the supersymmetry variations of the irreducible Weyl and vector multiplets [21]. We will ignore the hypermultiplets henceforth and take the coupled supersymmetry variations as a starting point.

Since the Weyl and vector multiplets are irreducible representations, the variations of the fields under supersymmetry transformations are independent of the action of the theory under consideration. As usual, a bosonic field configuration is supersymmetric when all fermion variations vanish. The supersymmetry conditions from the fermion variations are

$$
\begin{aligned}
\delta \psi_{\mu} & =\left(\mathcal{D}_{\mu}+\frac{1}{2} v^{a b} \gamma_{\mu a b}-\frac{1}{3} \gamma_{\mu} \gamma \cdot v\right) \epsilon=0 \\
\delta \Omega^{I} & =\left(-\frac{1}{4} \gamma \cdot F^{I}-\frac{1}{2} \gamma^{a} \partial_{a} M^{I}-\frac{1}{3} M^{I} \gamma \cdot v\right) \epsilon=0 \\
\delta \chi & =\left(D-2 \gamma^{c} \gamma^{a b} \mathcal{D}_{a} v_{b c}-2 \gamma^{a} \epsilon_{a b c d e} v^{b c} v^{d e}+\frac{4}{3}(\gamma \cdot v)^{2}\right) \epsilon=0
\end{aligned}
$$

where $\gamma \cdot T=\gamma_{a b} T^{a b}$ for a rank-2 tensor $T_{a b}$.

Once again, we wish to emphasize that the above variations are independent of the action of the theory. Indeed, this is the whole point of retaining the auxiliary fields. Consequently, the equations (2.2) serve as the supersymmetry conditions in the presence of higher-derivative terms.

\subsection{The two-derivative action}

After gauge fixing to Poincare supergravity the two-derivative Lagrangian constructed

5 We suppress the $S U(2)$ indices of $V_{\mu}$ and all fermionic variables because they play no role in our work. 
from the Weyl multiplet and $n_{V}$ vector multiplets reads

$$
\begin{aligned}
\mathcal{L}_{0}= & -\frac{1}{2} D-\frac{3}{4} R+v^{2}+\mathcal{N}\left(\frac{1}{2} D-\frac{1}{4} R+3 v^{2}\right)+2 \mathcal{N}_{I} v^{a b} F_{a b}^{I} \\
& +\mathcal{N}_{I J}\left(\frac{1}{4} F_{a b}^{I} F^{J a b}+\frac{1}{2} \partial_{a} M^{I} \partial^{a} M^{J}\right)+\frac{1}{24 e} c_{I J K} A_{a}^{I} F_{b c}^{J} F_{d e}^{K} \epsilon^{a b c d e} .
\end{aligned}
$$

One can integrate out the auxiliary fields $D$ and $v_{a b}$ by solving their equations of motion and substituting the solutions back into (2.3). This yields the familiar $N=2$ Lagrangian arising from the compactification of eleven-dimensional supergravity on a Calabi-Yau manifold with intersection numbers $c_{I J K}$ [29].

The functions defining the scalar manifold are

$$
\mathcal{N}=\frac{1}{6} c_{I J K} M^{I} M^{J} M^{K}, \quad \mathcal{N}_{I}=\partial_{I} \mathcal{N}=\frac{1}{2} c_{I J K} M^{J} M^{K}, \quad \mathcal{N}_{I J}=c_{I J K} M^{K},
$$

where $I, J, K=1, \ldots, n_{V}$. At the two-derivative level, the $D$ equation of motion imposes the constraint $\mathcal{N}=1$ defining real special geometry. However, higher derivative corrections make the geometry of the scalar moduli space more complicated.

\subsection{The four-derivative action}

A particular four-derivative term is special in that its coefficient is determined by M5brane anomaly cancellation via anomaly inflow [30]. This is the mixed gauge-gravitational Chern Simons term

$$
e \mathcal{L}_{\mathrm{CS}}=\frac{c_{2 I}}{24 \cdot 16} \epsilon_{a b c d e} A^{I a} R^{b c f g} R_{f g}^{d e} .
$$

It is believed that all four derivative terms are related to this term by supersymmetry [11]. The supersymmetric completion of the term (2.5) was derived in [11]. The bosonic terms are

$$
\begin{aligned}
\mathcal{L}_{1} & =\frac{c_{2 I}}{24}\left(\frac{1}{16 e} \epsilon_{a b c d e} A^{I a} C^{b c f g} C^{d e}{ }_{f g}+\frac{1}{8} M^{I} C^{a b c d} C_{a b c d}+\frac{1}{12} M^{I} D^{2}+\frac{1}{6} F^{I a b} v_{a b} D\right. \\
& +\frac{1}{3} M^{I} C_{a b c d} v^{a b} v^{c d}+\frac{1}{2} F^{I a b} C_{a b c d} v^{c d}+\frac{8}{3} M^{I} v_{a b} \hat{\mathcal{D}}^{b} \hat{\mathcal{D}}_{c} v^{a c} \\
& +\frac{4}{3} M^{I} \hat{\mathcal{D}}^{a} v^{b c} \hat{\mathcal{D}}_{a} v_{b c}+\frac{4}{3} M^{I} \hat{\mathcal{D}}^{a} v^{b c} \hat{\mathcal{D}}_{b} v_{c a}-\frac{2}{3 e} M^{I} \epsilon_{a b c d e} v^{a b} v^{c d} \hat{\mathcal{D}}_{f} v^{e f} \\
& +\frac{2}{3 e} F^{I a b} \epsilon_{a b c d e} v^{c f} \hat{\mathcal{D}}_{f} v^{d e}+e^{-1} F^{I a b} \epsilon_{a b c d e} v^{c}{ }_{f} \hat{\mathcal{D}}^{d} v^{e f} \\
& \left.-\frac{4}{3} F^{I a b} v_{a c} v^{c d} v_{d b}-\frac{1}{3} F^{I a b} v_{a b} v^{2}+4 M^{I} v_{a b} v^{b c} v_{c d} v^{d a}-M^{I}\left(v^{2}\right)^{2}\right)
\end{aligned}
$$

Here $C_{a b c d}$ is the Weyl tensor. The superconformal derivative is related to the usual derivative as $\hat{\mathcal{D}}_{\mu}=\mathcal{D}_{\mu}-b_{\mu}$. In our gauge the dilatational connection $b_{\mu}$ vanishes. However, its 
derivative does not vanish so the second superconformal covariant derivative is nontrivial, viz.

$$
v_{a b} \hat{\mathcal{D}}^{b} \hat{\mathcal{D}}_{c} v^{a c}=v_{a b} \mathcal{D}^{b} \mathcal{D}_{c} v^{a c}-\frac{2}{3} v^{a c} v_{c b} R_{a}{ }^{b}-\frac{1}{12} v_{a b} v^{a b} R
$$

The complete action (2.6) is evidently somewhat unwieldy. Fortunately, most terms play no role in our applications. For example, the parity-odd terms (proportional to $\epsilon_{a b c d e}$ ) vanish on our spacetimes. Their significance is that they fix the normalization through the anomaly term (2.5). We will ultimately need just the equations of motion for $D$ and for the gauge fields, and these involve just a few of the terms in (2.6).

\section{Magnetic solutions: strings with $\mathbf{A d S}_{3} \times S^{2}$ near horizon geometry}

Our strategy for finding regular solutions in the higher derivative theory is to first write an ansatz consistent with the assumed symmetries, and then demand unbroken supersymmetry. This part of the analysis proceeds the same whether we consider the two derivative or four derivative theory, and hence is quite manageable. Supersymmetry does not completely determine the solution, however — we also need to impose the Bianchi identity and the special geometry constraint, the latter coming from the $D$ equation of motion. Only at this last stage do we need specific information about the action.

\subsection{Ansatz: magnetic background}

We are interested in higher-derivative corrections to the supersymmetric black string solutions carrying magnetic charges $p^{I}$ studied in [31]. We assume translation invariance along the string, and spherical symmetry in the transverse directions. To make these symmetries explicit, we write our ansatz as

$$
d s^{2}=e^{2 U_{1}(r)}\left(d t^{2}-d x_{4}^{2}\right)-e^{-4 U_{2}(r)} d x^{i} d x^{i}, \quad d x^{i} d x^{i}=d r^{2}+r^{2} d \Omega_{2}^{2},
$$

where $i=1,2,3$. The two-forms $F^{I}$ and $v$ will be proportional to the volume form on $S^{2}$. We chose the vielbein as

$$
\begin{aligned}
e^{\hat{a}} & =e^{U_{1}} d x^{a}, \quad a=0,4, \\
e^{\hat{i}} & =e^{-2 U_{2}} d x^{i}, \quad i=1,2,3 .
\end{aligned}
$$

The non-trivial spin connections are

$$
\omega_{a}^{\hat{a} \hat{i}}=-e^{U_{1}+2 U_{2}} \partial_{i} U_{1}, \quad \omega_{k}^{\hat{i} \hat{j}}=2 \delta_{k}^{i} \partial_{j} U_{2}-2 \delta_{k}^{j} \partial_{i} U_{2} .
$$

\subsection{Supersymmetry conditions}

We start by analyzing the supersymmetry conditions (2.2) in the background (3.1). The supersymmetry parameter $\epsilon$ is constant along the string and obeys

$$
\gamma_{\hat{t} \hat{4}} \epsilon=-\epsilon
$$




\section{Gravitino variation}

We first analyze the gravitino variation (2.2)

$$
\delta \psi_{\mu}=\left(\mathcal{D}_{\mu}+\frac{1}{2} v^{a b} \gamma_{\mu a b}-\frac{1}{3} \gamma_{\mu} \gamma \cdot v\right) \epsilon=0 .
$$

For the background (3.1), the covariant derivative is

$$
\begin{aligned}
& \mathcal{D}_{a}=\partial_{a}-\frac{1}{2} e^{U_{1}+2 U_{2}} \partial_{i} U_{1} \gamma_{\hat{a} \hat{i}}, \\
& \mathcal{D}_{i}=\partial_{i}+\partial_{j} U_{2} \gamma_{\hat{i} \hat{j}} .
\end{aligned}
$$

Along the string, equation (3.5) simplifies to

$$
\left[-\frac{1}{2} e^{U_{1}+2 U_{2}} \partial_{i} U_{1} \gamma_{\hat{a} \hat{i}}+\frac{1}{6} e^{U_{1}} v_{\hat{i} \hat{j}} \gamma_{\hat{a} \hat{i} \hat{j}}\right] \epsilon=0 .
$$

It is convenient to use the projection (3.4) in the form

$$
\gamma_{\hat{i} \hat{j} \hat{k}} \epsilon=-\varepsilon_{i j k} \epsilon
$$

where $\varepsilon_{123}=1$. Then

$$
\gamma_{\hat{i} \hat{j}} \epsilon=\gamma^{\hat{k}} \gamma_{\hat{i} \hat{j} \hat{k}} \epsilon=\varepsilon_{i j k} \gamma_{\hat{k}} \epsilon
$$

So 3.7) becomes

$$
\left[-\frac{1}{2} e^{U_{1}+2 U_{2}} \partial_{k} U_{1}+\frac{1}{6} e^{U_{1}} v_{\hat{i} \hat{j}} \varepsilon_{i j k}\right] \gamma_{\hat{a} \hat{k}} \epsilon=0,
$$

from which we can solve for the auxiliary field,

$$
v_{\hat{i} \hat{j}}=\frac{3}{2} e^{2 U_{2}} \varepsilon_{i j k} \partial_{k} U_{1},
$$

or in coordinate frame

$$
v_{i j}=\frac{3}{2} e^{-2 U_{2}} \varepsilon_{i j k} \partial_{k} U_{1} .
$$

Consider now the components of the gravitino variation along $x^{i}$,

$$
\left[\partial_{i}+\partial_{j} U_{2} \gamma_{\hat{i} \hat{j}}+\frac{1}{2} v_{\hat{j} \hat{k}}\left(\gamma_{i \hat{j} \hat{k}}-\frac{2}{3} \gamma_{i} \gamma_{\hat{j} \hat{k}}\right)\right] \epsilon=0
$$

The $v_{\hat{j} \hat{k}}$ terms split into a "radial" part where either $j, k$ is equal to $i$, and an "angular" part where $i \neq j \neq k$. Thus we have two conditions

$$
\begin{aligned}
& 0=\left(\partial_{i}-\frac{1}{6} \varepsilon_{i j k} e_{i}^{\hat{i}} v_{\hat{j} \hat{k}}\right) \epsilon, \\
& 0=\left(\partial_{j} U_{2} \varepsilon_{i j k}+\frac{2}{3} v_{\hat{i} \hat{k}} e_{i}^{\hat{i}}\right) \gamma_{\hat{k}} \epsilon,
\end{aligned}
$$


where there is no summation over $i$. The second equation leads to $U_{2}=U_{1}$, so we will drop the subscripts on $U$ from now on. The first equation then leads to

$$
\left(\partial_{i}-\frac{1}{2} \partial_{i} U\right) \epsilon=0
$$

and so the Killing spinor takes the form

$$
\epsilon=e^{U / 2} \epsilon_{0}
$$

where $\epsilon_{0}$ is some constant spinor.

It will be convenient to use cylindrical coordinates from now on. The metric takes the form

$$
d s^{2}=e^{2 U}\left(d t^{2}-d x_{4}^{2}\right)-e^{-4 U}\left(d r^{2}+r^{2} d \Omega_{2}^{2}\right),
$$

in terms of a single function $U(r)$. The coordinate frame expression (3.12) is a tensor statement on the 3-dimensional base space, where $\varepsilon_{a b c}$ is a completely anti-symmetric tensor with components $\pm \sqrt{g}$. So in cylindrical coordinates the auxiliary two-form is

$$
v_{\theta \phi}=\frac{3}{2} e^{-2 U} r^{2} \sin \theta \partial_{r} U, \quad v_{\hat{\theta} \hat{\phi}}=\frac{3}{2} e^{2 U} \partial_{r} U
$$

with other components vanishing due to spherical symmetry in the transverse space. The projection (3.4) in cylindrical coordinates can be written as

$$
\gamma_{\hat{r} \hat{\theta} \hat{\phi}} \epsilon=-\epsilon
$$

\section{Gaugino variation}

Evaluated on the magnetic background, the gaugino variation $\delta \Omega^{I}$ in (2.2) gives

$$
\left(\gamma_{\hat{\theta} \hat{\phi}} F^{I \hat{\theta} \hat{\phi}}+\gamma^{\hat{r}} e_{\hat{r}}^{r} \partial_{r} M^{I}+\frac{4}{3} M^{I} \gamma_{\hat{\theta} \hat{\phi}} v^{\hat{\theta} \hat{\phi}}\right) \epsilon=0 .
$$

Using (3.19) and solving for the field strength we get

$$
\begin{aligned}
F^{I \hat{\theta} \hat{\phi}} & =e^{2 U} \partial_{r} M^{I}-\frac{4}{3} M^{I} v^{\hat{\theta} \hat{\phi}} \\
& =\partial_{r}\left(M^{I} e^{-2 U}\right) e^{4 U} .
\end{aligned}
$$

In coordinate frame, (3.21) becomes

$$
F_{\theta \phi}^{I}=\partial_{r}\left(M^{I} e^{-2 U}\right) r^{2} \sin \theta
$$

This equation is the first hint of the expected attractor behavior: the flow of the scalars $M^{I}$ is completely determined by the magnetic field $F^{I}$. 


\section{Auxiliary fermion variation}

The last supersymmetry variation to solve is $\delta \chi=0$. Neglecting the $\epsilon$-terms since we look for parity invariant solutions, this condition is

$$
\left(D-2 \gamma^{c} \gamma^{a b} \mathcal{D}_{a} v_{b c}+\frac{4}{3}(\gamma \cdot v)^{2}\right) \epsilon=0 .
$$

The relevant components of the covariant derivative of $v$ for the contraction in (3.23) are

$$
\mathcal{D}_{\theta} v_{r \phi}=\mathcal{D}_{\phi} v_{\theta r}=-\Gamma_{\theta r}^{\theta} v_{\theta \phi}, \mathcal{D}_{r} v_{\theta \phi}=\partial_{r} v_{\theta \phi}-2 \Gamma_{\theta r}^{\theta} v_{\theta \phi}
$$

with

$$
\Gamma_{\theta r}^{\theta}=\Gamma_{\phi r}^{\phi}=-2 \partial_{r} U+\frac{1}{r}
$$

Then, the second term in (3.23) becomes

$$
\begin{aligned}
\gamma^{c} \gamma^{a b} \mathcal{D}_{a} v_{b c} & =e_{\hat{r}}^{r} e_{\hat{\theta}}^{\theta} e_{\hat{\phi}}^{\phi}\left(-4 \mathcal{D}_{\theta} v_{r \phi}+2 \mathcal{D}_{r} v_{\theta \phi}\right) \gamma^{\hat{\gamma} \hat{\theta} \hat{\phi}} \\
& =2 \frac{e^{6 U}}{r^{2} \sin \theta} \partial_{r} v_{\theta \phi} \gamma^{\hat{r} \hat{\theta} \hat{\phi}} \\
& =\frac{3}{2} e^{6 U} \nabla^{2}\left(e^{-2 U}\right) \gamma_{\hat{r} \hat{\theta} \hat{\phi}}
\end{aligned}
$$

with $\nabla^{2}=\partial_{i} \partial_{i}=r^{-2} \partial_{r}\left(r^{2} \partial_{r}\right)$ due to spherical symmetry. Inserting (3.26) in (3.23) we have

$$
\left(D-3 e^{6 U} \nabla^{2}\left(e^{-2 U}\right) \gamma_{\hat{r} \hat{\theta} \hat{\phi}}-\frac{16}{3}\left(v_{\hat{\theta} \hat{\phi}}\right)^{2}\right) \epsilon=0,
$$

where we used

$$
(\gamma \cdot v)^{2}=-4\left(v_{\hat{\theta} \hat{\phi}}\right)^{2} .
$$

Using the projection (3.19) and substituting the auxiliary field (3.18) into (3.27) we find

$$
\begin{aligned}
D & =-3 e^{6 U} \nabla^{2}\left(e^{-2 U}\right)+\frac{16}{3}\left(v_{\hat{\theta} \hat{\phi}}\right)^{2} \\
& =3 e^{6 U}\left(-\nabla^{2}\left(e^{-2 U}\right)+4 e^{-2 U}(\nabla U)^{2}\right) \\
& =6 e^{4 U} \nabla^{2} U .
\end{aligned}
$$

What we have found so far is that supersymmetry demands a metric of the form (3.17), an auxiliary two tensor of the form (3.18), the gauge field strengths (3.22), and the auxiliary D-field (3.29). All told the entire solution is now specified in terms of the functions $M^{I}$ and $U$ which are not fixed by supersymmetry alone. 


\subsection{Equations of motion}

Having exhausted the implications of unbroken supersymmetry, we now need to use information from the equations of motion.

\section{Maxwell's equations}

Any specific string solution is parameterized by the values of the magnetic charges as measured by surface integrals at infinity. These in turn determine the gauge fields in the interior via the Maxwell equations.

We first consider the equation of motion

$$
\partial_{\theta}\left(\sqrt{g} \frac{\partial \mathcal{L}}{\partial F_{\theta \phi}^{I}}\right)=0
$$

Spherical symmetry implies that the expression in parenthesis is a function of $r$ only, hence (3.30) is satisfied identically for any field strength $F_{\theta \phi}^{I}=\mathcal{F}^{I}(r) \sin \theta$. Thus we get no new information from this equation of motion.

In the magnetic case the nontrivial condition arises from the Bianchi identity $d F^{I}=0$. The point is that the expression (3.21) for $F^{I}$ determined from supersymmetry is not automatically a closed form. Therefore, the Bianchi identity

$$
\partial_{r} F_{\theta \phi}^{I}=\partial_{r}\left(r^{2} \partial_{r}\left(M^{I} e^{-2 U}\right)\right) \sin \theta=0
$$

is nontrivial. Physically, this is because supersymmetry is consistent with any extended distribution of magnetic charges, while here we are demanding the absence of charge away from the origin. The equation (3.31) integrates to

$$
r^{2} \partial_{r}\left(M^{I} e^{-2 U}\right)=-\frac{p^{I}}{2}
$$

where $p^{I}$ is the quantized magnetic charge carried by $F^{I}$. We note that the field strength

$$
F^{I}=-\frac{p^{I}}{2} \epsilon_{2}
$$

does not get modified after including higher derivatives since it is topological.

The solutions to (3.32) are harmonic functions on the three-dimensional base space. We are just interested in the simplest solution

$$
M^{I} e^{-2 U}=H^{I}=M_{\infty}^{I}+\frac{p^{I}}{2 r},
$$

with $M_{\infty}^{I}$ the value of $M^{I}$ in the asymptotically flat region where $U=0$. 


\section{$D$ equation}

So far, by imposing the conditions for supersymmetry and integrating the Bianchi identity, we have been able to write our solution in terms of one unknown function $U(r)$. To determine this remaining function we use the equation of motion for the auxiliary field $D$. Inspecting (2.3) and (2.6) we see that the only D-dependent terms in the Lagrangian are

$$
\mathcal{L}_{D}=\frac{1}{2}(\mathcal{N}-1) D+\frac{c_{2 I}}{24}\left(\frac{1}{12} M^{I} D^{2}+\frac{1}{6} F^{I a b} v_{a b} D\right)
$$

Therefore, the equation of motion for $D$ is

$$
\mathcal{N}=1-\frac{c_{2 I}}{72}\left(F_{a b}^{I} v^{a b}+M^{I} D\right)
$$

Inserting the gauge-field (3.22), the auxiliary field (3.18), and the D-field (3.29) gives

$$
e^{-6 U}=\frac{1}{6} c_{I J K} H^{I} H^{J} H^{K}+\frac{c_{2 I}}{24}\left(\nabla H^{I} \nabla U+2 H^{I} \nabla^{2} U\right)
$$

Here $H^{I}$ are the harmonic functions defined in (3.34) and we used

$$
\mathcal{N}=\frac{1}{6} c_{I J K} H^{I} H^{J} H^{K} e^{6 U}
$$

The D constraint (3.37) is now an ordinary differential equation that determines $U(r)$. Its solution specifies the entire geometry and all the matter fields.

We can solve (3.37) exactly in the near horizon region. This case corresponds to vanishing integration constants in (3.34) so that

$$
H^{I}=\frac{p^{I}}{2 r}
$$

Then (3.37) gives

$$
e^{-6 U}=\frac{1}{8 r^{3}}\left(p^{3}+\frac{1}{12} c_{2} \cdot p\right)=\frac{\ell_{S}^{3}}{r^{3}},
$$

where $p^{3}=\frac{1}{6} c_{I J K} p^{I} p^{J} p^{K}$. The geometry in this case is $\operatorname{AdS}_{3} \times S^{2}$ with the scale $\ell_{S}$ in agreement with our previous work [21].

The asymptotically flat solutions to (3.37) cannot in general be found in closed form. In the following two subsections we discuss an approximate solution and an example of numerical integration. 


\subsection{Corrected geometry for large black strings}

One way to find solutions to (3.37) is by perturbation theory. This strategy captures the correct physics when the solution is regular already in the leading order theory, i.e. for large black strings. Accordingly, the starting point is the familiar solution

$$
e^{-6 U_{0}}=\frac{1}{6} c_{I J K} H^{I} H^{J} H^{K},
$$

to the two-derivative theory. This solves (3.37) with $c_{2 I}=0$.

Although $c_{2 I}$ is not small it will be multiplied by terms that are of higher order in the derivative expansion. It is therefore meaningful to expand the full solution to (3.37) in the form

$$
e^{-6 U}=e^{-6 U_{0}}+c_{2 I} \varepsilon^{I}+\frac{1}{2} c_{2 I} c_{2 J} \varepsilon^{I J}+\ldots,
$$

where $\varepsilon^{I}(r), \varepsilon^{I J}(r), \ldots$ determine the corrected geometry with increasing precision.

Inserting (3.42) in (3.37) and keeping only the terms linear in $c_{2 I}$ we find the first order correction 6

$$
\varepsilon^{I}=\frac{1}{24}\left(\nabla H^{I} \nabla U_{0}+2 H^{I} \nabla^{2} U_{0}\right) .
$$

Iterating, we find the second order correction

$$
\varepsilon^{I J}=-\frac{1}{72}\left(\nabla H^{I} \nabla\left(e^{6 U_{0}} \varepsilon^{J}\right)+2 H^{I} \nabla^{2}\left(e^{6 U_{0}} \varepsilon^{J}\right)\right),
$$

where the first order correction $\varepsilon^{I}$ is given by (3.43). Higher orders can be computed similarly. In summary, we find that starting from a smooth solution to the two-derivative theory we can systematically and explicitly compute the higher order corrections. The series is expected to be uniformly convergent.

In the near horizon limit (3.39) the full solution (3.40) is recovered exactly when taking the leading correction (3.43) into account. As indicated in (3.40) the effect of the higher derivative corrections is to expand the sphere by a specific amount (which is small for large charges). The perturbative solution gives approximate expressions for the corrections also in the bulk of the solution. Numerical analysis indicates that the corrections remain positive so at any value of the isotropic coordinate $r$ the corresponding sphere is expanded by a specific amount.

\subsection{Fundamental strings}

One of the main motivations for developing higher derivative corrections is their potential to regularize geometries that are singular in the lowest order supergravity approximation [4, 23, 24,66]. This is the situation for small strings, by which we mean charge configurations satisfying $p^{3}=\frac{1}{6} c_{I J K} p^{I} p^{J} p^{K}=0$.

\footnotetext{
${ }^{6}$ It is understood that the correction $\varepsilon^{I}$ is only defined in the combination $c_{2 I} \varepsilon^{I}$.
} 
A particularly important example of a small string is when the Calabi-Yau is $K 3 \times T^{2}$ and the only magnetic charge that is turned on is the one corresponding to an $M 5$-brane wrapping the $K 3$. The resulting $5 \mathrm{D}$ string is then dual, via IIA-heterotic duality, to the fundamental heterotic string [32,33].

Let $M^{1}$ be the single modulus on the torus and $M^{i}$ be the moduli of $K 3$ where $i=2, \ldots, 23$. The charge configuration of interest specifies the harmonic functions as

$$
\begin{aligned}
H^{1} & =M_{\infty}^{1}+\frac{p^{1}}{2 r}, \\
H^{i} & =M_{\infty}^{i}, \quad i=2, \ldots, 23 .
\end{aligned}
$$

The only nonvanishing intersection numbers are $c_{1 i j}=c_{i j}$ where $c_{i j}$ is the intersection matrix for $K 3$. We choose $M_{\infty}^{i}$ consistent with

$$
\mathcal{N} e^{-6 U}=\frac{1}{6} c_{I J K} H^{I} H^{J} H^{K}=H^{1}
$$

The master equation (3.37) now becomes

$$
H^{1}=e^{-6 U}-\left[\partial_{r} H^{1} \partial_{r} U+2 H^{1} \frac{1}{r^{2}} \partial_{r}\left(r^{2} \partial_{r} U\right)\right],
$$

where we used $c_{2}(K 3)=24$ and $c_{2 i}=0$. We can write this more explicitly as

$$
1+\frac{p^{1}}{2 r}=e^{-6 U}-2\left(1+\frac{p^{1}}{2 r}\right) U^{\prime \prime}-\frac{4}{r}\left(1+\frac{3 p^{1}}{8 r}\right) U^{\prime},
$$

where primes denote derivatives with respect to $r$.

In our units distance $r$ is measured in units of the 5D Planck length. The parameter $p^{1}$ is a pure number counting the fundamental strings. We take $p^{1} \gg 1$ so as to have an expansion parameter. We will analyze the problem one region at a time.

\section{The $\mathbf{A d S}_{3} \times S^{2}$-region}

This is the leading order behavior close to the string. According to our near horizon solution (3.40) we expect the precise asymptotics

$$
e^{-6 U} \rightarrow \frac{\ell_{S}^{3}}{r^{3}} \quad, r \rightarrow 0
$$

where the $S^{2}$-radius is given by

$$
\ell_{S}=\left(\frac{p^{1}}{4}\right)^{1 / 3}
$$


Since we assume $p^{1} \gg 1$ this is still much larger than the 5D Planck scale. The modulus describing the volume of the internal $T^{2}$ is

$$
M^{1}=\frac{p^{1}}{2 \ell_{S}}=2^{-1 / 3}\left(p^{1}\right)^{2 / 3}
$$

which also corresponds to the length scale $\left(p^{1}\right)^{1 / 3}$.

\section{The near-string region}

We next seek a solution in the entire range $r \ll p^{1}$ which includes the scale (3.50) but reaches further out. In fact, it may be taken to be all of space in a scaling limit where $p^{1} \rightarrow \infty$.

In the near string region (3.48) reduces to

$$
\frac{p^{1}}{2 r}=e^{-6 U}-\frac{p^{1}}{r} U^{\prime \prime}-\frac{3 p^{1}}{2 r^{2}} U^{\prime} .
$$

We can scale out the string number $p^{1}$ by substituting

$$
e^{-6 U(r)}=\frac{p^{1}}{4 r^{3}} e^{-6 \Delta(r)}
$$

which amounts to

$$
U(r)=\frac{1}{2} \ln \frac{r}{\ell_{S}}+\Delta(r)
$$

This gives

$$
\Delta^{\prime \prime}+\frac{3}{2 r} \Delta^{\prime}+\frac{1}{4 r^{2}}\left(1-e^{-6 \Delta}\right)+\frac{1}{2}=0,
$$

which describes the geometry in the entire region $r \ll p^{1}$. The asymptotic behavior at small $r$ is

$$
\Delta(r)=-\frac{1}{13} r^{2}+\frac{3}{(13)^{3}} r^{4}+\cdots
$$

Since $\Delta(r) \rightarrow 0$ smoothly as $r \rightarrow 0$ we have an analytical description of the approach to the $\mathrm{AdS}_{3} \times S^{2}$ region.

The asymptotic behavior for large $r$ is also smooth. Expanding in $u=\frac{1}{r}$ we find

$$
\Delta(r)=-\frac{1}{6} \ln \left(2 r^{2}\right)-\frac{1}{36 r^{2}}+\cdots .
$$

It is straightforward to solve (3.55) numerically. Figure 1 shows the curve that interpolates between the asymptotic forms (3.56) and (3.57). The oscillatory behavior in the intermediate region is characteristic of higher derivative theories. We comment in more detail below. 

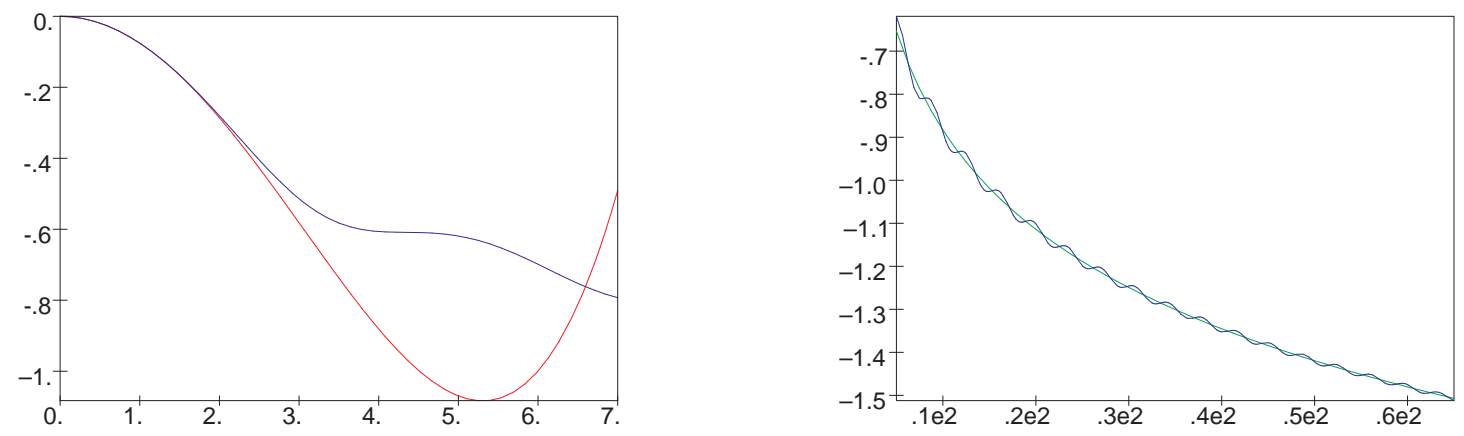

Fig. 1: Analytical and numerical results for $\Delta(r)$ in the near string region, $r \ll p^{1}$. In both plots the blue curve is given by solving (3.55) numerically. Left: the numerical solution close to the string with the approximate solution (3.56) given in red. Right: the numerical solution further away with the approximate solution (3.57) given in green. The plots have overlapping values of $r$ but different scales.

In the original variable $U(r)$ the approximation (3.57) gives

$$
e^{-6 U}=\frac{p^{1}}{2 r}\left(1+\frac{1}{6 r^{2}}+\ldots\right)
$$

for large $r$. The leading behavior agrees with the near string behavior $e^{-6 U}=H^{1} \sim \frac{p^{1}}{2 r}$ familiar from the description of a fundamental string in two-derivative supergravity. In the full theory this singular region is replaced by a smooth geometry described by $\Delta(r)$.

\section{The approach to asymptotically flat space}

We still need to analyze the region where $r$ is large, meaning $r \sim p^{1}$ or larger. Although standard two-derivative supergravity is expected to describe this region it is instructive to consider the possible corrections.

In the asymptotic region the full equation (3.47) simplifies to

$$
1+\frac{p^{1}}{2 r}=e^{-6 U}-2\left(1+\frac{p^{1}}{2 r}\right) U^{\prime \prime}
$$

Terms with explicit factors of $1 / r$ were neglected but we kept derivatives with respect to $r$, to allow for structure on Planck scale even though $r \sim p^{1} \gg 1$. Changing variables as

$$
e^{-6 U}=\left(1+\frac{p^{1}}{2 r}\right) e^{-6 W}
$$

we find

$$
W^{\prime \prime}=\frac{1}{2}\left(e^{-6 W}-1\right) \simeq-3 W
$$


The expansion for small $W$ is justified because (3.58) imposes the boundary condition $W \rightarrow 0$ for $r \ll p^{1}$.

The solution $W=0$ expected from supergravity is in fact a solution to (3.61) but there are also more general solutions of the form

$$
W=A \sin (\sqrt{3} r+\delta)
$$

The amplitude of this solution is undamped, so it is not really an intrinsic feature of the localized string solution we consider. Instead it is a property of fluctuations about flat space, albeit an unphysical one. The existence of such spurious solutions is a wellknown feature of theories with higher derivatives, and is related to the possibility of field redefinitions [34,23,24]. In the present context the issue is that other variables such as $\tilde{W}=\left(\nabla^{2}-3\right) W$ exhibit no spurious solutions. It would be interesting to make this interpretation of the spurious solutions more explicit. It would also be interesting to understand possible relations of our fundamental string solutions with the picture proposed in 35.

\section{Electric solutions: black holes with $\mathbf{A d S}_{2} \times S^{3}$ near horizon geometry}

We now consider the case of electrically charged, spherically symmetric solutions. We follow the same strategy as in the analysis of the magnetic solutions: we start from an ansatz with the desired symmetry, then use the supersymmetry conditions (2.2) to relate various functions in the ansatz, and finally impose appropriate equations of motion to obtain the full solution. The solutions we study are the higher-derivative corrected versions of those in [36].

In the electric case we start with a metric of the form

$$
d s^{2}=e^{4 U_{1}(x)} d t^{2}-e^{-2 U_{2}(x)} d x^{i} d x^{i}
$$

where $i=1 \ldots 4$. This gives the vielbein

$$
e^{\hat{t}}=e^{2 U_{1}} d t, \quad e^{\hat{i}}=e^{-U_{2}} d x^{i}
$$

and spin connections

$$
\omega_{t}^{\hat{t} \hat{i}}=-2 \partial_{i} U_{1} e^{2 U_{1}+U_{2}}, \quad \omega_{k}^{\hat{i} \hat{j}}=\partial_{j} U_{2} \delta_{k}^{i}-\partial_{i} U_{2} \delta_{k}^{j}
$$

In this paper we limit ourselves to spherically symmetric solutions for which

$$
v^{\hat{i} \hat{j}}=0
$$




\subsection{Supersymmetry conditions}

We now make the conditions (2.2) imposed by supersymmetry explicit for our electric ansatz.

\section{Gravitino variation}

We begin with constraints from the gravitino variation, the first equation in (2.2). The temporal part of the gravitino variation reads

$$
\left[\partial_{t}+\frac{1}{2} \omega_{t}^{\hat{t} \hat{i}} \gamma_{\hat{t} \hat{i}}+\frac{1}{2} v^{a b}\left(\gamma_{t a b}-\frac{2}{3} \gamma_{t} \gamma_{a b}\right)\right] \epsilon=0 .
$$

We assume that the Killing spinor is time-independent and satisfies the projection

$$
\gamma_{\hat{t}} \epsilon=-\epsilon .
$$

Inserting (4.4) we find

$$
\left(\frac{1}{2} \omega_{t}^{\hat{t} \hat{i}}-\frac{2}{3} e_{t}^{\hat{t}} v^{\hat{t} \hat{i}}\right) \gamma_{\hat{i}} \epsilon=0
$$

which implies

$$
v_{\hat{t} \hat{i}}=\frac{3}{2} \partial_{i} U_{1} e^{U_{2}} .
$$

The spatial part of the gravitino variation is

$$
\left[\partial_{i}+\frac{1}{4} \omega_{i}^{\hat{k} \hat{j}} \gamma_{\hat{k} \hat{j}}+v^{\hat{\hat{t}} \hat{j}}\left(\gamma_{i \hat{t} \hat{j}}-\frac{2}{3} \gamma_{i} \gamma_{\hat{t} \hat{j}}\right)\right] \epsilon=0 .
$$

Substituting the formulae for the connection and using projection (4.6) yields

$$
\left[\partial_{i}+\frac{1}{2} \partial_{j} U_{2} \gamma_{\hat{i} \hat{j}}+v^{\hat{t} \hat{j}}\left(\gamma_{i \hat{j}}-\frac{2}{3} \gamma_{i} \gamma_{\hat{j}}\right)\right] \epsilon=0 .
$$

The radial $(\hat{i}=\hat{j})$ and the angular $(\hat{i} \neq \hat{j})$ terms have a different form. Therefore they must vanish separately so that

$$
\begin{aligned}
& \left(\partial_{i}+\frac{2}{3} v^{\hat{t} \hat{i}} e_{i}^{\hat{i}}\right) \epsilon=0, \\
& \frac{1}{2} \partial_{j} U_{2}+\frac{1}{3} v^{\hat{\hat{t}} \hat{j}} e_{i}^{\hat{i}}=0 .
\end{aligned}
$$

Inserting $v_{\hat{t} \hat{i}}$ from (4.8) into the second equation we find that $U_{1}(x)=U_{2}(x)$. Therefore we will drop the index on the function $U(x)$ from now on. The first equation in (4.11) gives the form of the Killing spinor

$$
\epsilon=e^{U(x)} \epsilon_{0},
$$


where $\epsilon_{0}$ is a constant spinor.

In summary, the gravitino equation determines the form of the Killing spinor (4.12), the auxiliary field

$$
v_{\hat{t} \hat{i}}=\frac{3}{2} \partial_{i} e^{U}, \quad v_{t i}=\frac{3}{2} e^{2 U} \partial_{i} U,
$$

and simplifies the metric from (4.1) to

$$
d s^{2}=f^{2} d t^{2}-f^{-1} d x^{i} d x^{i},
$$

where $f=e^{2 U(x)}$.

\section{Gaugino variation}

We next analyze the gaugino variation, the second equation in (2.2). Noting that only $v_{\hat{t} \hat{i}}$ are non-vanishing, and using the projection (4.6), we have

$$
\left(-\frac{1}{4} \gamma \cdot F^{I}-\frac{1}{2} \gamma^{a} \partial_{a} M^{I}-\frac{2}{3} M^{I} v^{\hat{t} \hat{i}} \gamma_{\hat{i}}\right) \epsilon=0 .
$$

This requires that the scalars are time-independent and that the only non-zero components of the field strengths are $F^{\hat{t} \hat{i}}$. This in turn gives the condition

$$
-\frac{1}{2} F^{I \hat{t} \hat{i}}+\frac{1}{2} e_{\hat{i}}^{i} \partial_{i} M^{I}-\frac{2}{3} M^{I} v^{\hat{t} \hat{i}}=0 .
$$

Inserting the auxiliary field (4.13) and switching to a coordinate frame we find

$$
\frac{1}{2} e^{-2 U} F_{i t}^{I}-\frac{1}{2} \partial_{i} M^{I}-M^{I} \partial_{i} U=0 .
$$

Reorganizing, we have

$$
F_{i t}^{I}=\partial_{i}\left(e^{2 U} M^{I}\right)
$$

which can be integrated to

$$
A_{t}^{I}=e^{2 U} M^{I} .
$$

This equation captures the characteristic feature of attractor flows: the scalars follow the electric potentials along the entire radial flow.

\section{Auxiliary fermion variation}

Imposing $\delta \chi=0$ results in the condition

$$
\left(D-2 \gamma^{c} \gamma^{a b} \mathcal{D}_{a} v_{b c}-2 \gamma^{a} \epsilon_{a b c d e} v^{b c} v^{d e}+\frac{4}{3}(\gamma \cdot v)^{2}\right) \epsilon=0 .
$$


The third term vanishes in the spherically symmetric case and the fourth term can be evaluated as

$$
\frac{4}{3}(\gamma \cdot v)^{2}=\frac{16}{3} \delta^{i j} v_{\hat{t} \hat{i}} v_{\hat{t} \hat{j}}=12 e^{2 U} \partial_{i} U \partial_{i} U .
$$

For the covariant derivative we need the non-vanishing Christoffel symbols

$$
\Gamma_{t t}^{i}=2 e^{6 U} \partial_{i} U, \quad \Gamma_{t i}^{t}=2 \partial_{i} U, \quad \Gamma_{i j}^{k}=\left(\delta_{i j} \partial_{k}-\delta_{i k} \partial_{j}-\delta_{k j} \partial_{i}\right) U .
$$

and the auxiliary field (4.13). This gives the only non-vanishing component of the covariant derivative as

$$
\mathcal{D}_{i} v_{t j}=\frac{3}{2} e^{2 U}\left(\partial_{i} \partial_{j} U+2 \partial_{i} U \partial_{j} U-\delta_{i j} \partial_{k} U \partial_{k} U\right)
$$

so that

$$
\gamma^{c} \gamma^{a b} \mathcal{D}_{a} v_{b c}=\gamma^{i} \gamma^{j t} \mathcal{D}_{j} v_{t i}=-\gamma_{\hat{t}} \delta^{i j} \mathcal{D}_{j} v_{t i}=-\frac{3}{2} e^{2 U} \gamma_{\hat{t}}\left(\partial_{i} \partial_{i} U-2 \partial_{i} U \partial_{i} U\right)
$$

After applying the projection (4.6) on the supersymmetry parameter $\epsilon$, the condition (4.20) from the variation of the auxiliary fermion now becomes

$$
\begin{aligned}
D & =3 e^{2 U}\left(\partial_{i} \partial_{i} U-2 \partial_{i} U \partial_{i} U\right)-12 e^{2 U} \partial_{i} U \partial_{i} U \\
& =3 e^{2 U}\left(\nabla^{2} U-6(\nabla U)^{2}\right) .
\end{aligned}
$$

We have now exhausted the supersymmetry conditions (2.2). As a result we have found (4.13), (4.19), and (4.25) which determine $v_{a b}, A_{t}^{I}$, and $D$ in terms of the scalar moduli $M^{I}$ and the metric function $U(x)$. These remaining functions are not determined by supersymmetry alone. Instead we must now turn to the equations of motion.

\subsection{Maxwell equations}

The black hole solutions we seek are defined by conserved electric charges with respect to each gauge field. For a given charge, Gauss' law determines the radial dependence of the electric field as follows.

Neglecting the Chern-Simons terms, which do not contribute to spherically symmetric solutions, the F-dependent terms in the Lagrangian are

$$
\begin{aligned}
\mathcal{L}_{F}= & 2 \mathcal{N}_{I} v^{a b} F_{a b}^{I}+\frac{1}{4} \mathcal{N}_{I J} F_{a b}^{I} F^{J a b} \\
& +\frac{c_{2 I}}{24}\left(\frac{1}{6} F_{a b}^{I} v^{a b} D+\frac{1}{2} F^{I a b} C_{a b c d} v^{c d}-\frac{4}{3} F^{I a b} v_{a c} v^{c d} v_{d b}-\frac{1}{3} F^{I a b} v_{a b} v^{2}\right) .
\end{aligned}
$$

The Maxwell equations

$$
\mathcal{D}_{\mu}\left(\frac{\partial \mathcal{L}}{\partial F_{\mu \nu}^{I}}\right)=\frac{1}{\sqrt{g}} \partial_{\mu}\left(\sqrt{g} \frac{\partial \mathcal{L}}{\partial F_{\mu \nu}^{I}}\right)=0
$$


are equivalent to the statement $\partial_{r} q_{I}=0$, where $q_{I}$ are the conserved electric charges

$$
q_{I}=-\frac{1}{4 \pi^{2}} \int_{S^{3}} \sqrt{g} \frac{\partial \mathcal{L}}{\partial F_{t r}^{I}}=-\frac{1}{2} e^{-2 U} r^{3} \mathcal{E}_{I}^{r} .
$$

The canonical momenta are

$$
\mathcal{E}_{I}^{i}=\frac{\partial \mathcal{L}}{\partial F_{t i}^{I}}=4 \mathcal{N}_{I} v^{t i}+\mathcal{N}_{I J} F^{J t i}+\frac{c_{2 I}}{24}\left(\frac{1}{3} v^{t i} D+2 C^{t i t j} v_{t j}-\frac{8}{3} v^{t j} v_{j t} v^{t i}-\frac{2}{3} v^{t i} v^{2}\right) .
$$

We need to make this expression more explicit. First, let us define moduli with lower indices ast

$$
M_{I}=\frac{1}{2} \mathcal{N}_{I J} M^{J}
$$

In the context of Calabi-Yau compactification of M-theory the $M_{I}$ are volumes of fourcycles dual to the two-cycles with volume $M^{I}$. At any rate, the definitions (2.4) of the various scalar functions now imply

$$
\mathcal{N}_{I J} \partial_{i} M^{J}=\partial_{i} M_{I} .
$$

We now find

$$
4 \mathcal{N}_{I} v^{t i}+\mathcal{N}_{I J} F^{J t i}=e^{2 U} \partial_{i}\left[e^{-2 U} M_{I}\right],
$$

due to (4.13) and (4.18). It is straightforward to cast the remaining terms in (4.29) in this form as well, by using (4.13), (4.18), (4.25), along with

$$
C^{i t j t}=-2 \partial_{i} \partial_{j} U-6 \partial_{i} U \partial_{j} U+\frac{3}{2} \delta_{i j}(\nabla U)^{2}+\frac{1}{2} \delta_{i j} \nabla^{2} U .
$$

After the dust has settled we find

$$
\mathcal{E}_{I}^{i}=e^{2 U} \partial_{i}\left[e^{-2 U} M_{I}-\frac{c_{2 I}}{8}(\nabla U)^{2}\right] .
$$

We now see that the Maxwell equation $\partial_{r} q_{I}=0$ becomes

$$
\nabla^{2}\left[e^{-2 U} M_{I}-\frac{c_{2 I}}{8}(\nabla U)^{2}\right]=0
$$

where $\nabla^{2}$ denotes the Laplacian on flat $\mathbb{R}^{4}$. Solutions are thus a set of harmonic functions on this space. In this paper we consider the single center solutions

$$
e^{-2 U} M_{I}-\frac{c_{2 I}}{8}(\nabla U)^{2}=H_{I}=M_{I}^{\infty}+\frac{q_{I}}{r^{2}},
$$

where the integration constants $M_{I}^{\infty}$ are the moduli at infinity, and the $q_{I}$ are the same charges as appear in (4.28).

7 This notation is actually redundant because $M_{I}=\mathcal{N}_{I}$. 


\subsection{Completing the electric solution: the $D$ equation}

At this point we have used supersymmetry to specify the entire solution in terms of the functions $M^{I}$ and $U$, and we have determined $M^{I}$ by integrating Gauss' Law. Therefore, we need only one more constraint to find the complete solution. For this we consider the equation of motion for the auxiliary field $D$.

Starting from the Lagrangian $\mathcal{L}_{0}+\mathcal{L}_{1}$ given in (2.3) and (2.6), the terms that depend on the $D$-field are

$$
\mathcal{L}_{D}=\frac{1}{2}(\mathcal{N}-1) D+\frac{1}{24} c_{2 I}\left(\frac{1}{12} M^{I} D^{2}+\frac{1}{6} F^{I a b} v_{a b} D\right)
$$

Varying with respect to $D$ we find

$$
\mathcal{N}-1+\frac{c_{2 I}}{72}\left(M^{I} D+F^{I a b} v_{a b}\right)=0
$$

From (4.25), (4.18), and (4.13), this becomes

$$
\mathcal{N}-1+\frac{c_{2 I}}{24} e^{2 U}\left(\left(\nabla^{2} U-4(\nabla U)^{2}\right) M^{I}+\nabla U \nabla M^{I}\right)=0 .
$$

In the absence of higher derivative terms this equation simply reads $\mathcal{N}=1$. Since $\mathcal{N}=$ $\frac{1}{6} c_{I J K} M^{I} M^{J} M^{K}$ this amounts to an algebraic constraint on the scalar manifold. The general equation with higher derivatives included is much more complicated. To be explicit, recall that the $M^{I}$ are determined in terms of $M_{I}$ by the relation

$$
M_{I}=\frac{1}{2} c_{I J K} M^{J} M^{K}
$$

and the $M_{I}$ in turn are given by

$$
\left.M_{I}=e^{2 U}\left(H_{I}+\frac{c_{2 I}}{8}(\nabla U)^{2}\right)\right), \quad H_{I}=M_{I}^{\infty}+\frac{q_{I}}{r^{2}} .
$$

The condition (4.39) is thus a nonlinear, second order, ordinary differential equation for $U(r)$. But note that to write this equation explicitly requires inverting (4.40) to find $M^{I}$, which cannot be done until $c_{I J K}$ have been specified. 8 Once this has been done, the resulting differential equation typically requires a numerical treatment.

8 One also should be alert to the fact that the inversion of 4.40 may not be unique, which raises some interesting issues. Some explicit examples in related contexts can be found in [37,38]. 


\subsection{Near Horizon Geometry}

It is instructive to make the equations above more explicit in the near horizon region of the black hole. To do so take the integration constants $M_{I}^{\infty}=0$ and seek a solution with constant $M_{I}$ and $M^{I}$ related by (4.40). Then (4.41) gives $f=e^{2 U}=\frac{r^{2}}{\ell_{S}^{2}}$ and

$$
\ell_{S}^{2} M_{I}=q_{I}+\frac{1}{8} c_{2 I} .
$$

The notation $\ell_{S}$ was chosen with some foresight. Indeed, the change of variables $r^{2}=\ell_{S}^{3} / 2 z$ bring the geometry (4.14) into the standard form

$$
d s^{2}=\frac{\ell_{S}^{2}}{4 z^{2}}\left(d t^{2}-d z^{2}\right)-\ell_{S}^{2} d \Omega_{3}^{2},
$$

which we recognize as $\mathrm{AdS}_{2} \times S^{3}$ with $S^{3}$ radius $\ell_{S}$ and $\mathrm{AdS}_{2}$ radius $\ell_{A}=\frac{1}{2} \ell_{S}$.

In the near horizon region the $D$ equation (4.39) is an algebraic constraint

$$
\mathcal{N}=\frac{1}{6} c_{I J K} M^{I} M^{J} M^{K}=1+\frac{1}{12 \ell_{S}^{2}} c_{2 I} M^{I} .
$$

If we write the inversion equation (4.40) in terms of the rescaled variables

$$
\hat{M}^{I} \equiv \ell_{S} M^{I}
$$

it becomes

$$
\frac{1}{2} c_{I J K} \hat{M}^{J} \hat{M}^{K}=q_{I}+\frac{1}{8} c_{2 I} .
$$

This is an algebraic equation that determines $\hat{M}^{I}$ as functions of the charges $q_{I}$ and the numbers $c_{I J K}$ and $c_{2 I}$. Given such a solution, $\hat{M}^{I}=\hat{M}^{I}\left(q_{J}\right)$, the constraint (4.44) gives the scale of the geometry

$$
\ell_{S}^{3}=\frac{1}{6} c_{I J K} \hat{M}^{I} \hat{M}^{J} \hat{M}^{K}-\frac{1}{12} c_{2 I} \hat{M}^{I},
$$

where the right hand side is a function of the charges alone. Finally, 4.45) and (4.46) give the physical moduli in the near horizon region as

$$
\begin{aligned}
& M_{I}=\frac{q_{I}+\frac{1}{8} c_{2 I}}{\left(\frac{1}{6} c_{I J K} \hat{M}^{I} \hat{M}^{J} \hat{M}^{K}-\frac{1}{12} c_{2 I} \hat{M}^{I}\right)^{2 / 3}}, \\
& M^{I}=\frac{\hat{M}^{I}}{\left(\frac{1}{6} c_{I J K} \hat{M}^{I} \hat{M}^{J} \hat{M}^{K}-\frac{1}{12} c_{2 I} \hat{M}^{I}\right)^{1 / 3}} .
\end{aligned}
$$

The expressions (4.46)-(4.48) completely specify the near horizon geometry of the 5D black hole. 


\subsection{Example: $K 3 \times T^{2}$}

In order to illustrate how our final expression (4.39) determines the entire radial dependence of the solution, we next present a numerical solution in the special case of $K 3 \times T^{2}$. As in the analogous magnetic example (section 3.5) we let $M^{1}$ be the single modulus on the torus and $M^{i}$ be the moduli of $K 3$, where $i=2 \ldots 23$. The only nonvanishing intersection numbers are then $c_{1 i j} \equiv c_{i j}$, where $c_{i j}$ is the intersection matrix for $K 3$, with inverse $c^{i j}$.

From $M_{I}=\frac{1}{2} c_{I J K} M^{J} M^{K}$, we find

$$
M_{1}=\frac{1}{2} c_{i j} M^{i} M^{j}, \quad M_{i}=c_{i j} M^{j} M^{1},
$$

We easily invert this to obtain the $M^{I}$ as functions of the $M_{I}$

$$
M^{1}=\sqrt{\frac{c^{i j} M_{i} M_{j}}{2 M_{1}}}, \quad M^{i}=c^{i j} M_{j} \sqrt{\frac{2 M_{1}}{c^{k l} M_{k} M_{l}}} .
$$

The Chern class $c_{2 I}$ is calculated on the 4-cycle Poincare dual to the $I$-th 2-cycle. Therefore, the $c_{2 i}$ vanish, leaving only $c_{2,1}=c_{2}(K 3)=24$.

Substituting (4.41) into (4.50) to yields

$$
M^{1}=\left(\frac{e^{2 U} c^{i j} H_{i} H_{j}}{2 H_{1}+6\left(U^{\prime}\right)^{2}}\right)^{1 / 2}, \quad M^{i}=\left(\frac{e^{2 U} c^{i j} H_{i} H_{j}}{2 H_{1}+6\left(U^{\prime}\right)^{2}}\right)^{-1 / 2} e^{2 U} c^{i j} H_{j}
$$

where primes denote derivatives with respect to $r$. The special geometry constraint (4.39) is

$$
\frac{1}{2} c_{i j} M^{i} M^{j} M^{1}-1+e^{2 U}\left[\left(U^{\prime \prime}+\frac{3}{r} U^{\prime}-4 U^{\prime 2}\right) M^{1}+U^{\prime}\left(M^{1}\right)^{\prime}\right]=0 .
$$

The problem is now to insert (4.51) into (4.52) and solve for $U(r)$.

This is straightforward to solve numerically, given specific choices of charges. Consider a small black hole, $q_{1}=0$ with $q_{2}=q_{3}=1, c^{23}=1$. We also assume $H=H_{2}=H_{3}=1+\frac{1}{r^{2}}$ are the only harmonic functions not equal to unity. Then (4.52) becomes

$$
H U^{\prime \prime}+\left(1+3\left(U^{\prime}\right)^{2}\right)\left[U^{\prime} r^{-1}\left(3+\frac{1}{r^{2}}\right)+H\right]-e^{-3 U}\left(1+3\left(U^{\prime}\right)^{2}\right)^{3 / 2}=0 .
$$

The boundary conditions are fixed by matching to the desired small $r$ behavior

$$
e^{-2 U} \sim \frac{\ell_{S}^{2}}{r^{2}}
$$

with $\ell_{S}=3^{-1 / 6}$. The result of the numerical analysis is the $U(r)$ shown in Figure 2. It exhibits the same kind of oscillations seen in [23,24] and discussed in the end of section 3.5 . 


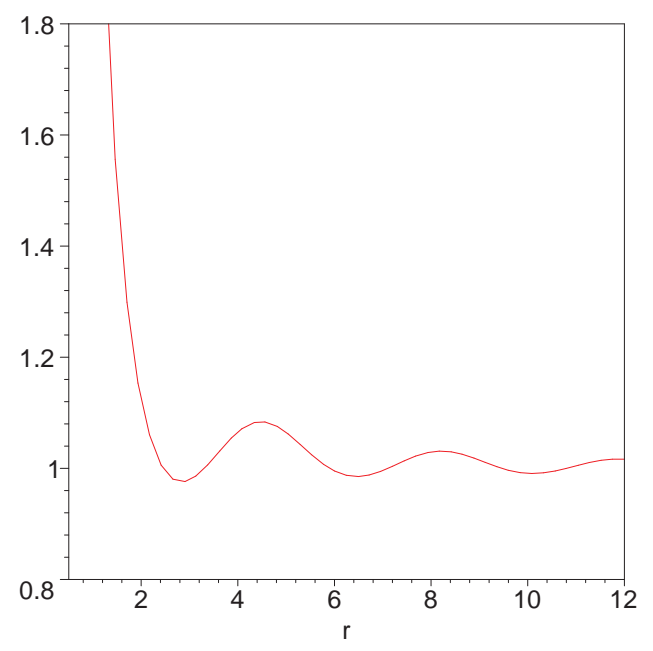

Fig. 2: Numerical solution of (4.53). The plot displays $e^{-2 U(r)}$ for small $r$.

\section{Entropy of electrically charged black holes}

\subsection{Entropy function}

We now turn to the computation of the entropy of the electric black hole solutions. Due to the higher derivative corrections, the relevant formula is Wald's generalized expression for the entropy as a surface integral over the horizon [39]. Generally it can be laborious to integrate Wald's entropy density, but the extremal black holes we consider have a near horizon $\mathrm{AdS}_{2}$ factor, and for such black holes the problem reduces to evaluating an "entropy function" at its extremum [7]. The entropy function is the Legendre transformation of the action with respect to the electric charges. In our conventions, the precise expression is

$$
S=\pi \ell_{A}^{2} \ell_{S}^{3}\left(F_{t r}^{I} \frac{\partial \mathcal{L}}{\partial F_{t r}^{I}}-\mathcal{L}\right)
$$

where $\mathcal{L}$ is the Lagrangian density evaluated in the near horizon $\operatorname{AdS}_{2} \times S^{3}$ geometry.

\subsection{Near horizon supersymmetry}

To proceed we need to evaluate $\mathcal{L}=\mathcal{L}_{0}+\mathcal{L}_{1}$, and its derivative with respect to the field strength. This task is greatly simplified by taking advantage of the conditions resulting from enhancement of supersymmetry in the near horizon region. This means there is no need to impose any projector condition on the Killing spinor $\epsilon$, and so each term in the supersymmetry conditions (2.2) vanishes by itself, rather than balancing off other terms.

To see how this works, recall that the variation of the auxiliary fermion includes the term (4.9)

$$
\gamma^{c} \gamma^{a b} \mathcal{D}_{a} v_{b c}=-\frac{3}{2} e^{2 U} \gamma_{\hat{t}}\left(\frac{1}{r^{3}} \partial_{r}\left(r^{3} \partial_{r} U\right)-2\left(\partial_{r} U\right)^{2}\right)
$$


Taking this to vanish we immediately find a metric function of the form

$$
e^{2 U}=\frac{r^{2}}{\ell_{S}^{2}}
$$

for some $\ell_{S}$. This is recognized as the metric function describing $\operatorname{AdS}_{2} \times S^{3}$ (4.43) with the scales of the constituent spaces related as

$$
\ell_{A}=\frac{1}{2} \ell_{S}
$$

With the geometry in hand, the $D$-field (4.25) determined from the variation of the auxiliary fermion becomes

$$
D=3 e^{2 U}\left(\partial^{2} U-6(\partial U)^{2}\right)=-\frac{12}{\ell_{S}^{2}},
$$

and the auxiliary two-form (4.13) determined from the gravitino variation becomes

$$
v_{t r}=\frac{3}{2} e^{2 U} \partial_{r} U=\frac{3 r}{2 \ell_{S}^{2}} .
$$

The gaugino variation (4.15) shows that the moduli $M^{I}$ are constants in the near horizon region. It also gives the field strengths (4.18) as

$$
F_{t r}^{I}=-\partial_{r}\left(e^{2 U} M^{I}\right)=-\frac{2 r}{\ell_{S}^{2}} M^{I} .
$$

Our general expression (4.28) for the electric charge can be written in the near horizon region as

$$
q_{I}=-\frac{1}{2} \ell_{S}^{2} r \frac{\partial \mathcal{L}}{\partial F_{t r}^{I}}
$$

Using (5.4), (5.7), and (5.8), the entropy function becomes

$$
S=\pi\left(\ell_{S} q \cdot M-\frac{\ell_{S}^{5}}{4} \mathcal{L}\right)
$$

\subsection{Evaluation of entropy function}

Up until now we have just used the supersymmetry variations, which are independent of the action. We now need to use details of the action. The first piece of information we need is the modified special geometry constraint (4.39) coming from the $D$ equation of motion. Using the near horizon field values found above we recover (4.44)

$$
\mathcal{N}-1-\frac{c_{2} \cdot M}{12 \ell_{S}^{2}}=0
$$


Next, we need to evaluate the Lagrangian density. Using the near horizon supersymmetry results, as well as $(5.10)$, we find for the two derivative Lagrangian $\mathcal{L}_{0}$,

$$
\mathcal{L}_{0}=\frac{4}{\ell_{S}^{2}}+\frac{1}{3} \frac{c_{2} \cdot M}{\ell_{S}^{4}}
$$

after some algebra. For the four derivative Lagrangian $\mathcal{L}_{1}$,

$$
\mathcal{L}_{1}=-\frac{1}{2} \frac{c_{2} \cdot M}{\ell_{S}^{4}}
$$

after more algebra. Altogether

$$
\mathcal{L}=\mathcal{L}_{0}+\mathcal{L}_{1}=\frac{4}{\ell_{S}^{2}}-\frac{1}{6} \frac{c_{2} \cdot M}{\ell_{S}^{4}}
$$

giving the entropy function

$$
S=\pi\left(\ell_{S} q \cdot M-\ell_{S}^{3}+\frac{1}{24} \ell_{S} c_{2} \cdot M\right)
$$

At this stage we could evaluate $S$ by inserting the values for $\ell_{S}$ and $M^{I}$ obtained from our explicit solutions, but it is more instructive to proceed by extremizing the entropy function. This also serves as a useful consistency check on our results.

The problem consists of extremizing $S$ with respect to $\ell_{S}$ and $M^{I}$, while holding fixed $q_{I}$ and imposing the constraint (5.10). We therefore add in a Lagrange multiplier and write

$$
S=\pi\left(\ell_{S} q \cdot M-\ell_{S}^{3}+\frac{1}{24} \ell_{S} c_{2} \cdot M+\lambda\left(\mathcal{N}-1-\frac{c_{2} \cdot M}{12 \ell_{S}^{2}}\right)\right) .
$$

Extremizing gives

$$
\begin{aligned}
& 0=q \cdot M-3 \ell_{S}^{2}+\frac{1}{24} c_{2} \cdot M+\frac{1}{6} \lambda \frac{c_{2} \cdot M}{\ell_{S}^{3}}, \\
& 0=\ell_{S} q_{I}+\frac{1}{24} \ell_{S} c_{2 I}+\lambda \mathcal{N}_{I}-\frac{1}{12} \lambda \frac{c_{2 I}}{\ell_{S}^{2}}, \\
& 0=\mathcal{N}-1-\frac{c_{2} \cdot M}{12 \ell_{S}^{2}} .
\end{aligned}
$$

We can solve for $\lambda$ as follows. Contract the second equation with $\frac{1}{\ell_{S}} M^{I}$, subtract it from the first, and use the third to eliminate $\mathcal{N}$. This gives

$$
\lambda=-\ell_{S}^{3}
$$

Before continuing, we can use these equations to rewrite the entropy in a suggestive form. Using (5.17), and the first and third equations of (5.16), we insert into (5.15) to get

$$
S=2 \pi \mathcal{N} \ell_{S}^{3}
$$


This is the same formula as we would find in the two derivative theory, except that in that case we would have $\mathcal{N}=1$. From the higher dimensional point of view the condition $\mathcal{N}=1$ corresponds to fixing the $C Y_{3}$ to have unit volume. More generally, if we continue to think of $\mathcal{N}$ as the volume, then we see that (5.18) is precisely $S=A / 4 G$, with $A$ being the horizon area in 11 dimensions. However, one should perhaps not take this too seriously, since in the presence of higher derivatives the metric, and hence the horizon area, are subject to field redefinition ambiguities.

From (5.17) and the middle equation of (5.16) we can now solve for $\mathcal{N}_{I}$ as

$$
\ell_{S}^{2} \mathcal{N}_{I}=q_{I}+\frac{1}{8} c_{2 I}
$$

This agrees with our previous result (4.42), which we obtained by integrating Gauss' law and matching onto charges defined in the asymptotically flat region. The agreement is a nontrivial check on the consistency of our method (and the accuracy of our algebra).

We are now ready to find the entropy. Introducing the rescaled moduli

$$
\hat{M}^{I}=\ell_{S} M^{I}
$$

as in 4.45, the entropy (5.18) becomes simply

$$
S=2 \pi\left(\frac{1}{6} c_{I J K} \hat{M}^{I} \hat{M}^{J} \hat{M}^{K}\right) .
$$

The rescaled moduli can be found by solving (5.19) written in the form

$$
\frac{1}{2} c_{I J K} \hat{M}^{J} \hat{M}^{K}=\hat{q}_{I}
$$

where the shifted charge is defined as

$$
\hat{q}_{I}=q_{I}+\frac{1}{8} c_{2 I}
$$

The solution to (5.22) will take the form $\hat{M}^{I}\left(\hat{q}_{I}\right)$ which we then insert in (5.21) to find the entropy as function of the charges.

The value of $\ell_{S}$ can be computed by solving the special geometry constraint 5.10 ) from which we recover our previous result (4.47) for $\ell_{S}$. However, we do not actually need $\ell_{S}$ to find the entropy, because the factors of $\ell_{S}$ were scaled away when arriving at the entropy formula (5.21).

The computation of the entropy in terms of the $\hat{q}_{I}$ is almost insensitive to the detailed form of the action. All we need is (5.21) and (5.22) which could be derived using just the conditions due to enhancement of supersymmetry. To get the right shift in the definition 
(5.23) of $\hat{q}_{I}$, though, we need to use some information about the action, such as the $D$ equation of motion. Assuming we know this shift, we see that if we know the black hole entropy in the two derivative theory, then the corrected entropy is obtained simply by replacing the charges by the shifted charges.

Strictly speaking, the regime of validity of our computation only extends to terms first order in $c_{2 I}$, since we only considered the addition of four-derivative terms to the action. A priori, $2+2 n$ derivative terms in the action will contribute at the same order as any $\left(c_{2 I}\right)^{n}$ terms. In the case of black holes / strings with near horizon geometry $\mathrm{AdS}_{3} \times S^{2}$, one can use anomalies and supersymmetry to prove that the four derivative action in fact gives the exact expression for the large momentum behavior of the entropy [6,22]. In the present case it is also tempting to conjecture that the regime of validity extends beyond the first order terms, at least in some cases.

\subsection{Comparison with other results}

\section{Comparison with 4D black holes and the topological string}

In 25] an entropy formula for 5D black holes was conjectured, based on 4D results and the topological string partition function. The entropy is given by

$$
S=\mathcal{F}-\phi^{I} \frac{\partial \mathcal{F}}{\partial \phi^{I}}
$$

with

$$
\mathcal{F}=-\frac{1}{\pi^{2}}\left(D_{I J K} \phi^{I} \phi^{J} \phi^{K}-\frac{\pi^{2}}{6} c_{2 I} \phi^{I}\right)
$$

This yields

$$
S=\frac{2}{\pi^{2}} D_{I J K} \phi^{I} \phi^{J} \phi^{K}
$$

To convert to our notation, use

$$
D_{I J K}=\frac{1}{6} c_{I J K}, \quad \phi^{I}=\pi \hat{M}^{I}
$$

so that the entropy becomes

$$
S=2 \pi\left(\frac{1}{6} c_{I J K} \hat{M}^{I} \hat{M}^{J} \hat{M}^{K}\right),
$$

in precise agreement with (5.21).

On the other hand, in [25] the electric charges are

$$
q_{I}=-\frac{\partial \mathcal{F}}{\partial \phi^{I}}=\frac{1}{2} c_{I J K} \hat{M}^{J} \hat{M}^{J}-\frac{1}{6} c_{2 I}
$$


which is equivalent to

$$
\hat{q}_{I}=q_{I}+\frac{1}{6} c_{2 I}
$$

This is to be compared with (5.23). Thus, when expressed in terms of the $q_{I}$ our entropy formula does not agree with [25]. The reason for this mismatch is explained in detail in [40]. The shift (5.30) gives the wrong result for the 5D black hole since it includes an extra charge induced by the Taub-NUT geometry.

We also note that the authors of [25] performed a 5D supergravity computation keeping only the Gauss-Bonnet like term. This computation yielded a different discrepancy, which is not surprising since the full action contains many more terms at this order.

\section{$K 3 \times T^{2}$ black holes}

In general we have to invert (5.22) to express the entropy in terms of the electric charges $q_{I}$. This can be done explicitly when $c_{I J K}$ are the intersection numbers for $K 3 \times T^{2}$. This is basically the same problem we solved in (4.50), with solution

$$
\hat{M}^{1}=\sqrt{\frac{c^{i j} \hat{q}_{i} \hat{q}_{j}}{2 \hat{q}_{1}}}, \quad \hat{M}^{i}=\sqrt{\frac{2 \hat{q}_{1}}{c^{k l} \hat{q}_{k} \hat{q}_{l}}} c^{i j} q_{j} .
$$

The entropy is then

$$
S=\pi \sqrt{2 \hat{q}_{1} c^{i j} \hat{q}_{i} \hat{q}_{j}}=\pi \sqrt{2\left(q_{1}+3\right) c^{i j} q_{i} q_{j}} .
$$

A small black hole corresponds to taking $q_{1}=0$, such that the M2-branes lie entirely within $K 3$. The higher derivative terms give a finite size horizon to this would-be singular charge configuration.

\section{Elliptically fibred Calabi-Yau black holes}

There is no known microscopic description of black holes made from wrapping M2branes on a generic Calabi-Yau. However, in [26] Vafa proposed such a description for an elliptically fibred Calabi-Yau. This proposal yields a correction to the entropy that has the right form to match with a four derivative term in five dimensions. We can use our results to check that the coefficient also agrees.

Consider M-theory on a Calabi-Yau 3-fold $K$ realized as an elliptic fiber $E$ over a base space $B$. Wrap $M 2$ branes along a two-cycle $[C]+n[E]$, i.e. one that has components along the fiber and also along $C \subset B$. In [26] it was argued that the relevant moduli space

is the symmetric product $\operatorname{Sym}^{n}(\hat{C})$, where $\hat{C}$ is an elliptically fibred four manifold with base $C$. BPS states are then computed from the cohomology of $\hat{C}$ in the standard fashion. The cohomology leads to the entropy formula

$$
S=\pi \sqrt{2 n\left(C \cdot C+3 c_{1}(C)+2\right)}
$$


where the intersection products refer to the base $B$. This formula is valid for large $n$. Now, if we follow [26] and use $c_{1}(C)=\frac{1}{12} c_{2}(\hat{C})$, we see that the leading order correction to the entropy corresponds to the shift

$$
C \cdot C \rightarrow C \cdot C+\frac{1}{4} c_{2}(\hat{C})
$$

This matches the leading order shift obtained from (5.23),

$$
\hat{q} \cdot \hat{q} \approx q \cdot q+\frac{1}{4} c_{2} \cdot q
$$

\section{Acknowledgments:}

The work of PK and JD is supported in part by NSF grant PHY-0456200. The work of FL and AC is supported by DOE under grant DE-FG02-95ER40899.

\section{Appendix A. Conventions}

We briefly summarize our conventions. The metric signature is mostly minus $\eta_{a b}=$ $\operatorname{diag}(+,-,-,-,-) .9$ Covariant derivatives of spinors are defined as

$$
\mathcal{D}_{\mu}=\partial_{\mu}+\frac{1}{4} \omega_{\mu}^{a b} \gamma_{a b}
$$

where $\omega^{a b}$ are the spin-connection one forms related to the vielbein through the Cartan equation

$$
d e^{a}+\omega_{b}^{a} \wedge e^{b}=0
$$

Our convention for the curvature is

$$
R_{\mu \nu \kappa}^{\lambda}=\partial_{\kappa} \Gamma_{\mu \nu}^{\lambda}-\partial_{\nu} \Gamma_{\mu \kappa}^{\lambda}+\Gamma_{\mu \nu}^{\sigma} \Gamma_{\kappa \sigma}^{\lambda}-\Gamma_{\mu \kappa}^{\sigma} \Gamma_{\nu \sigma}^{\lambda}
$$

The scalar curvature is then, e.g., $R=\frac{p(p-1)}{\ell_{A}^{2}}-\frac{q(q-1)}{\ell_{S}^{2}}$ for $\operatorname{AdS}_{p} \times S^{q}$. The Weyl tensor is given by

$$
C_{a b c d}=R_{a b c d}-\frac{2}{3}\left(g_{a[c} R_{d] b}-g_{b[c} R_{d] a}\right)+\frac{1}{6} g_{a[c} g_{d] b} R .
$$

Anti-symmetric products of gamma-matrices are normalized so that $\gamma_{a b c d e}=\varepsilon_{a b c d e}$ where $\varepsilon_{01234}=1$.

Finally, we take $G_{5}=\frac{\pi}{4}$ and measure moduli in units of $2 \pi \ell_{11}$. In these units the charges are quantized (for review see [41]).

9 The signature is opposite to that in our previous paper [21. 


\section{References}

[1] K. Behrndt, G. Lopes Cardoso, B. de Wit, D. Lust, T. Mohaupt and W. A. Sabra, "Higher-order black-hole solutions in $\mathrm{N}=2$ supergravity and Calabi-Yau string backgrounds," Phys. Lett. B 429, 289 (1998) [arXiv:hep-th/9801081;;G. Lopes Cardoso, B. de Wit, D. Lust, T. Mohaupt, "Corrections to macroscopic supersymmetric blackhole entropy", Phys. Lett. B 451, 309 (1999) arXiv:hep-th/9812082. "Macroscopic entropy formulae and non-holomorphic corrections for supersymmetric black holes", Nucl. Phys. B 567, 87 (2000) arXiv:hep-th/9906094]; G. Lopes Cardoso, B. de Wit, J. Kappeli , T. Mohaupt "Stationary BPS solutions in $\mathrm{N}=2$ supergravity with $R^{2}$ interactions", JHEP 0012, 019 (2000) [arXiv:hep-th/0009234;

[2] H. Ooguri, A. Strominger and C. Vafa, "Black hole attractors and the topological string", Phys. Rev. D 70, 106007 (2004), arXiv:hep-th/0405146;

[3] A. Sen, "Black holes, elementary strings and holomorphic anomaly," JHEP 0507, 063 (2005) arXiv:hep-th/0502126]; "Entropy function for heterotic black holes," JHEP 0603, 008 (2006) arXiv:hep-th/0508042] ; B. Sahoo and A. Sen, "alpha' corrections to extremal dyonic black holes in heterotic string theory," JHEP 0701, 010 (2007) arXiv:hep-th/0608182.

[4] A. Dabholkar, "Exact counting of black hole microstates," Phys. Rev. Lett. 94, 241301 (2005) arXiv:hep-th/0409148.

[5] A. Dabholkar, F. Denef, G. W. Moore and B. Pioline, "Exact and asymptotic degeneracies of small black holes", arXiv:hep-th/0502157; "Precision counting of small black holes," JHEP 0510, 096 (2005) [arXiv:hep-th/0507014].

[6] P. Kraus and F. Larsen, "Microscopic black hole entropy in theories with higher derivatives," JHEP 0509, 034 (2005) arXiv:hep-th/0506176].

[7] A. Sen, "Black hole entropy function and the attractor mechanism in higher derivative gravity," JHEP 0509, 038 (2005) arXiv:hep-th/0506177.

[8] T. Mohaupt, "Black hole entropy, special geometry and strings," Fortsch. Phys. 49, 3 (2001) arXiv:hep-th/0007195.

[9] B. Pioline, "Lectures on black holes, topological strings and quantum attractors," Class. Quant. Grav. 23, S981 (2006) arXiv:hep-th/0607227.

[10] P. Kraus, "Lectures on black holes and the $\operatorname{AdS}(3) / \mathrm{CFT}(2)$ correspondence," arXiv:hepth/0609074.

[11] K. Hanaki, K. Ohashi and Y. Tachikawa, "Supersymmetric completion of an $\mathrm{R}^{* *} 2$ term in five-dimensional supergravity," arXiv:hep-th/0611329.

[12] I. Bena and P. Kraus, 'Microstates of the D1-D5-KK system," Phys. Rev. D 72, 025007 (2005) arXiv:hep-th/0503053; "Microscopic description of black rings in AdS/CFT," JHEP 0412, 070 (2004) arXiv:hep-th/0408186]. 
[13] H. Elvang, R. Emparan, D. Mateos and H. S. Reall, "Supersymmetric 4D rotating black holes from 5D black rings," JHEP 0508, 042 (2005) arXiv:hep-th/0504125].

[14] D. Gaiotto, A. Strominger and X. Yin, "New connections between 4D and 5D black holes," JHEP 0602, 024 (2006) arXiv:hep-th/0503217.

[15] D. Gaiotto, A. Strominger and X. Yin, "5D black rings and 4D black holes," JHEP 0602, 023 (2006) arXiv:hep-th/0504126.

[16] I. Bena, P. Kraus and N. P. Warner, "Black rings in Taub-NUT," Phys. Rev. D 72, 084019 (2005) arXiv:hep-th/0504142.

[17] K. Behrndt, G. Lopes Cardoso and S. Mahapatra, "Exploring the relation between 4D and 5D BPS solutions," Nucl. Phys. B 732, 200 (2006) arXiv:hep-th/0506251.

[18] A. Strominger and C. Vafa, "Microscopic Origin of the Bekenstein-Hawking Entropy," Phys. Lett. B 379, 99 (1996) arXiv:hep-th/9601029].

[19] J. C. Breckenridge, R. C. Myers, A. W. Peet and C. Vafa, "D-branes and spinning black holes," Phys. Lett. B 391, 93 (1997) arXiv:hep-th/9602065.

[20] A. Castro, J. L. Davis, P. Kraus and F. Larsen. In progress.

[21] A. Castro, J. L. Davis, P. Kraus and F. Larsen, "5D attractors with higher derivatives," arXiv:hep-th/0702072.

[22] P. Kraus and F. Larsen, "Holographic gravitational anomalies," JHEP 0601, 022 (2006) arXiv:hep-th/0508218.

[23] A. Dabholkar, R. Kallosh and A. Maloney, "A stringy cloak for a classical singularity," JHEP 0412, 059 (2004) arXiv:hep-th/0410076; V. Hubeny, A. Maloney and M. Rangamani, "String-corrected black holes," JHEP 0505, 035 (2005) arXiv:hepth/0411272.

[24] A. Sen, "How does a fundamental string stretch its horizon?," JHEP 0505, 059 (2005) arXiv:hep-th/0411255;

[25] M. Guica, L. Huang, W. Li and A. Strominger, "R**2 corrections for 5D black holes and rings," JHEP 0610, 036 (2006) arXiv:hep-th/0505188].

[26] C. Vafa, "Black holes and Calabi-Yau threefolds," Adv. Theor. Math. Phys. 2, 207 (1998) arXiv:hep-th/9711067.

[27] T. Kugo and K. Ohashi, "Supergravity tensor calculus in 5D from 6D," Prog. Theor. Phys. 104, 835 (2000) arXiv:hep-ph/0006231; T. Fujita and K. Ohashi, "Superconformal tensor calculus in five dimensions," Prog. Theor. Phys. 106, 221 (2001) arXiv:hep-th/0104130.

[28] E. Bergshoeff, S. Cucu, M. Derix, T. de Wit, R. Halbersma and A. Van Proeyen, "Weyl multiplets of $\mathrm{N}=2$ conformal supergravity in five dimensions," JHEP 0106, 051 (2001) arXiv:hep-th/0104113. "N = 2 supergravity in five dimensions revisited," Class. Quant. Grav. 21, 3015 (2004) [Class. Quant. Grav. 23, 7149 (2006)] arXiv:hepth/0403045]. 
[29] A. C. Cadavid, A. Ceresole, R. D'Auria and S. Ferrara, Phys. Lett. B 357, 76 (1995) arXiv:hep-th/9506144.

[30] M. J. Duff, J. T. Liu and R. Minasian, "Eleven-dimensional origin of string / string duality: A one-loop test," Nucl. Phys. B 452, 261 (1995) [arXiv:hep-th/9506126].

[31] A. H. Chamseddine and W. A. Sabra, "Calabi-Yau black holes and enhancement of supersymmetry in five dimensions," Phys. Lett. B 460, 63 (1999) arXiv:hepth/9903046].

[32] A. Sen, "String String Duality Conjecture In Six-Dimensions And Charged Solitonic Nucl. Phys. B 450, 103 (1995) arXiv:hep-th/9504027.

[33] J. A. Harvey and A. Strominger, "The Heterotic String Is A Soliton," Nucl. Phys. B 449, 535 (1995) [Erratum-ibid. B 458, 456 (1996)] arXiv:hep-th/9504047.

[34] B. Zwiebach, "Curvature Squared Terms And String Theories," Phys. Lett. B 156, 315 (1985).

[35] A. Giveon and D. Kutasov, "Fundamental strings and black holes," JHEP 0701, 071 (2007) arXiv:hep-th/0611062.

[36] W. A. Sabra, Mod. Phys. Lett. A 13, 239 (1998) arXiv:hep-th/9708103.

[37] M. Shmakova, "Calabi-Yau black holes," Phys. Rev. D 56, 540 (1997) arXiv:hepth/9612076].

[38] A. Chou, R. Kallosh, J. Rahmfeld, S. J. Rey, M. Shmakova and W. K. Wong, "Critical points and phase transitions in 5d compactifications of M-theory," Nucl. Phys. B 508, 147 (1997) arXiv:hep-th/9704142.

[39] R. M. Wald, "Black hole entropy is the Noether charge," Phys. Rev. D 48, 3427 (1993) arXiv:gr-qc/9307038. R. Wald, Phys. Rev. D 48 R3427 (1993); V. Iyer and R. M. Wald, "Some properties of Noether charge and a proposal for dynamical black hole entropy," Phys. Rev. D 50, 846 (1994) arXiv:gr-qc/9403028]. "A Comparison of Noether charge and Euclidean methods for computing the entropy of stationary black holes," Phys. Rev. D 52, 4430 (1995) arXiv:gr-qc/9503052.

[40] A. Castro, J. L. Davis, P. Kraus and F. Larsen, "Precision entropy of spinning black holes," arXiv:0705.1847 [hep-th].

[41] F. Larsen, "The attractor mechanism in five dimensions," arXiv:hep-th/0608191. 